European Association for the

Development of Renewable Energies,

Environment and Power Quality
International Conference on Renewable Energies and Power Quality (ICREPQ'09)

Valencia (Spain), 15th to 17th April, 2009

\title{
A Kriging-Based Partial Shading Analysis in a Large Photovoltaic Field for Energy Forecast
}

\author{
${ }^{1}$ Università degli Studi di Palermo \\ Dipartimento di Ingegneria Idraulica e Applicazioni Ambientali \\ (DIIAA) \\ Viale delle Scienze - 90128 PALERMO, ITALY \\ annalisadipiazza@hotmail.it
}

Annalisa Di Piazza ${ }^{1}$, Maria Carmela Di Piazza ${ }^{2}$, Member IEEE, Gianpaolo Vitale ${ }^{2}$, Member IEEE

\begin{abstract}
The use of kriging for the analysis of partial shading in photovoltaic (PV) fields is investigated. The considered method gives a spatial interpolation of the shadowing so that its distribution over all the PV field surface can be obtained on the basis of a limited number of experimental data. In all studied cases the kriging estimates matched the shadowing profile, also with a strongly reduced number of measured data. This approach also allows a cheaper and simpler characterization of the PV plant output power.

Keywords: Models and simulation of renewable energy sources; Photovoltaic array; Statistics; Kriging estimation.
\end{abstract}

\section{Introduction}

PV fields are composed of matrices of solar cells interconnected in series and parallel. Therefore the array performance is dependent on the behavior of the individual solar cells. This could be critical especially in non-ideal operating conditions, such as partial shading. Partial shading leads to a reduction of the output power. Moreover the occurrence of multiple local maxima, due to non uniform solar irradiation, can cause a failure of the MPPT control with a consequent considerable power loss due to the lack of the real MPP detection [1]. For this reason the correct experimental determination of solar irradiance distribution over a PV field is crucial. In this paper a new method based on a kriging regressor for obtaining an accurate estimate of irradiance distribution over a large PV array by a reduced number of irradiance sensors is proposed. The method spatially interpolates the irradiance values obtained by the reduced number of available sensors over the whole PV field area. So a nonuniform or varying irradiance and the resulting power capability of the PV system can be identified in a cheaper way and managing a smaller amount of experimental data.

\section{Features of the PV array}

The considered PV field is composed of 120 modules (Solar world- SW 160) each of them having a surface of $0.81 \mathrm{~m} \times 1.61 \mathrm{~m}$ and a rated power of $160 \mathrm{Wp}$. The total power of the plant is about $20 \mathrm{kWp}$. The strings are composed of 10 modules. Six single phase inverters are used for the grid connection of the PV field. Each inverter is supplied by two parallel connected strings, forming an array.

\author{
${ }^{2}$ Consiglio Nazionale delle Ricerche \\ Istituto di Studi sui Sistemi Intelligenti per l'Automazione \\ (ISSIA - CNR), sezione di Palermo, \\ Via Dante, 1290141 PALERMO, ITALY \\ TEL. +390916113513 FAX +390916113028 \\ mariacarmela.dipiazza@ieee.org gianpaolo.vitale@iieee.org
}

\section{Kriging theory}

Kriging is a statistical estimation technique for spatial interpolation of random quantities. The kriging method allows to obtain the quantity value at an unobserved location from observations of its value at nearby locations, being the unknown value obtained by a weighted mean of the available data [2]-[5].

The ordinary kriging formulation of the estimator is the following:

$\bar{z}\left(s_{0}\right)=\sum_{i=1}^{n} \lambda_{i} z\left(s_{i}\right)$

Eq. (1) means that kriging method finds a local estimate of the quantity at a specified location, $\mathrm{s}_{0}$. This estimate is a weighted average of the $\mathrm{N}$ adjacent observations, where the weighting coefficients, $\lambda_{\mathrm{i}}$, can be determined on the basis of the minimum estimation variance criterion [2].

\section{Data generation}

A function that gives a good approximation of the partial shading on a large PV field is the bidimensional Gaussian function [6]. Such a function is identified once two parameters, for each field dimension ( $x$ or $y$ ), are defined, i.e., the coordinates of the shading cloud centre and its width. Therefore the normalized shadowing, $Z$, over the $\mathrm{PV}$ field is described as:

$Z(x, y)=\exp \left(-\frac{\left(x-x_{c}\right)^{2}}{\sigma_{x}}-\frac{\left(y-y_{c}\right)^{2}}{\sigma_{y}}\right)$

where $\left(x_{c}, y_{c}\right)$ represent the coordinates of the centre of the shading cloud and $\sigma_{x}$ and $\sigma_{y}$ are its dimensions, respectively along $x$ and $y$ axes. It should be noted that $\mathrm{Z}\left(x_{c}, y_{c}\right)=1$ corresponds to the maximum shadowing. The spatial distribution of the solar irradiance is given by: $G(x, y)=\operatorname{Gmax}[1-\mathrm{Z}(x, y)]$

where Gmax is the solar radiation without shadowing. The following study cases are explored. Case 1: One shading cloud on the PV field surface with coordinates of the center $\mathrm{x}=5, \mathrm{y}=12$ and dimensions $\sigma_{\mathrm{x}}=5 \mathrm{~m}$ and $\sigma_{\mathrm{y}}=12$ $\mathrm{m}$. Case 2: Two partially superimposed shading clouds on the PV field surface. One cloud has the center coordinates $\mathrm{x}=5, \mathrm{y}=6$ and dimensions $\sigma_{\mathrm{x}}=10 \mathrm{~m}$ and $\sigma_{\mathrm{y}}=10 \mathrm{~m}$, the other has the center coordinates $\mathrm{x}=7.5$, $\mathrm{y}=12$ and dimensions $\sigma_{\mathrm{x}}=5 \mathrm{~m}$ and $\sigma_{\mathrm{y}}=5 \mathrm{~m}$. 


\section{Kriging processing}

The kriging processing of the shadowing data, previously illustrated, is carried out by the EasyKrig $\odot 3.0$ software. The kriging estimates of the shadowing distribution over the PV array are determined in the case 1 and in the case 2 by considering firstly the configuration with one irradiance sensor for each module (full configuration) then a configuration with sensors reduced up to the $25 \%$ (reduced configuration). In Figs 1 and 2 the obtained results are shown in terms of kriging maps. In all cases, results obtained by the reduced configuration are fitting with the ones registered when one sensor for each module is employed.

\section{Energy Forecast}

The obtained results are used for the evaluation of energy supplied by the PV field under partial shading conditions. This analysis is carried out with reference to the case 1 (see Fig. 3). The electrical characteristics of the PV modules are obtained by a modelling and a parameter identification technique, described in [7]. With uniform irradiance the P-V curves exhibit a unique maximum, while, under partial shading, these curves have multiple local maxima with a reduction of the whole available power. The I-V and P-V characteristics of the strings in array 4 are illustrated in Figs. 4 and 5: with solar irradiance measured with one sensor for each module and with solar irradiance measured with $25 \%$ of sensors and interpolated by kriging in the other modules. The goodness of the interpolating technique to find out the actual maximum PV output power, under partial shading conditions, is verified.
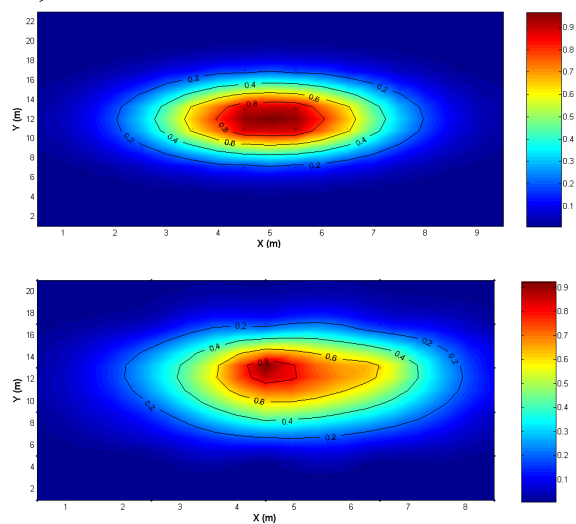

Fig.1. Kriging maps of the shadowing in case 1: full configuration of sensors.(upper plot); reduced configuration of sensors. (lower plot).
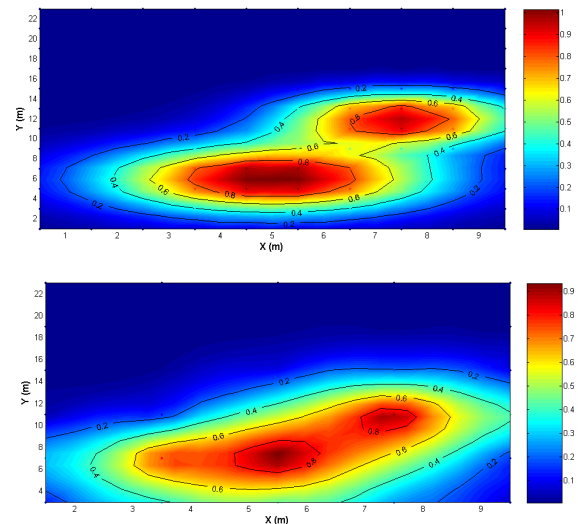

Fig.2. Kriging maps of the shadowing in case 2: full configuration of sensors.(upper plot); reduced configuration of sensors. (lower plot).

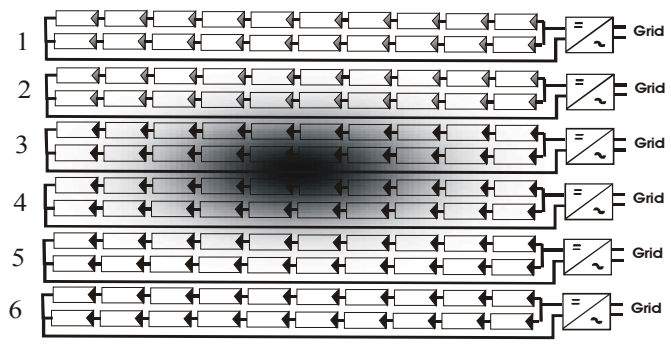

Fig.3. Partially shaded plant: case 1.
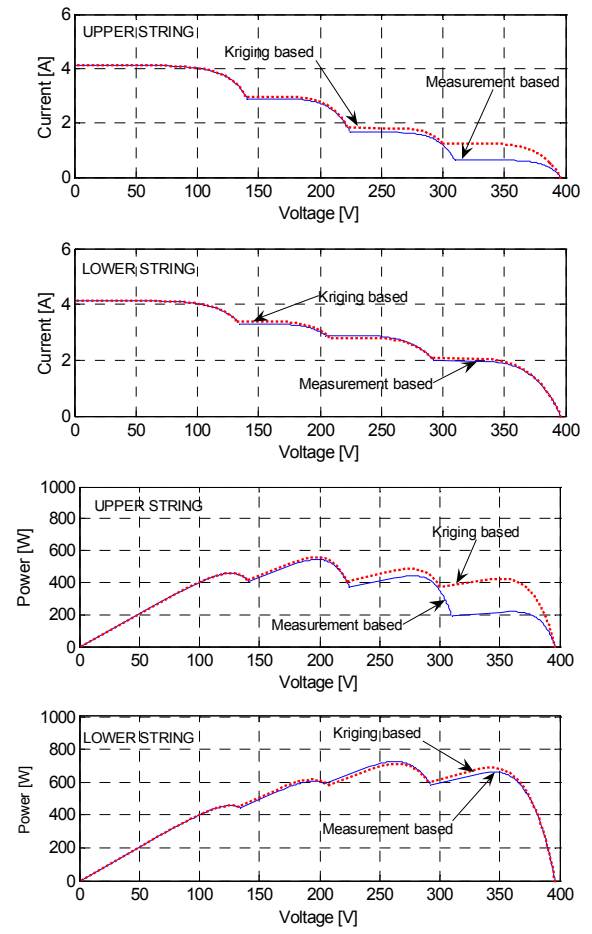

Fig.18. I-V characteristics of the upper and lower strings in array 4.

\section{Conclusion}

The spatial distribution of the shadowing over the surface of a large PV field is computed by means of the kriging interpolation method. The proposed approach allows the accurate estimation of the partial shading by using a very small number of experimental observations of solar irradiance. Therefore the method leads to a less expensive characterization of the PV plant output power and represents an useful tool for PV plants energy assessment.

\section{References}

[1] T. Esram, P. L. Chapman, "Comparison of photovoltaic array maximum power point tracking techniques", IEEE trans. Energy Conver., vol. 22, no.2, Jun. 2007.

[2] Deutsch, C. V and A. G. Journel, 1992. GSLIB: Geostatistical Software Library and User's Guide. Oxford University Press, Oxford. [3] Cressie, N (1993) Statistics for spatial data, Wiley, New York.

[4] X. Emery (2005) Simple and Ordinary Kriging Multigaussian Kriging for Estimating recovevearble Reserves, Mathematical Geology, v. 37, n. 3, pp. 295-319.

[5] Hanefi Bayraktar and F. Sezer. Turalioglu (2005) A Kriging-based approach for locating a sampling site in the assessment of air quality, SERRA, v.19, n.4, DOI 10.1007/s00477-005-0234-8, pp. 301-305.

[6] P. Erto "Probabilità e Statistica per le Scienze e l'Ingegneria", McGraw-Hill,2004.

[7] M.C. Di Piazza, C. Serporta, G. Vitale, "A DC/DC Converter Based Circuit Model for a Solar Photovoltaic Array", 21th European Photovoltaic Solar Energy Conference and Exibition, 4-8 September 2006, Dresda, Germany. 